\title{
Abdominal compartment syndrome and decompressive laparotomy in children: a 9-year single-center experience
}

\author{
Anthony di Natale ${ }^{1}$ (D) $\cdot$ Ueli Moehrlen ${ }^{1} \cdot$ Hannah Rachel Neeser ${ }^{1} \cdot$ Noëmi Zweifel $^{1} \cdot$ Martin Meuli $^{1}$. \\ Andrea Alexis Mauracher ${ }^{2}$. Barbara Brotschi ${ }^{3}$. Sasha Job Tharakan ${ }^{1}$
}

Accepted: 17 February 2020 / Published online: 28 February 2020

c) Springer-Verlag GmbH Germany, part of Springer Nature 2020

\begin{abstract}
Purpose Abdominal compartment syndrome (ACS) in children results in 100\% mortality if left untreated. Decompressive laparotomy (DL) is the only effective treatment if conservative medical therapies have failed. This study aims to determine the incidence of ACS among pediatric patients who underwent an emergency laparotomy (EL), to describe the effect of DL on clinical and laboratory parameters and, to make a better prediction on fatal outcome, to analyze variables and their association with mortality.

Methods This retrospective study includes 418 children up to the age of 16 years who underwent EL between January 2010 and December 2018 at our tertiary pediatric referral center. ACS was defined according to the latest guidelines of the World Society of the Abdominal Compartment Syndrome.

Results Fourteen patients had emergency DL for ACS. $6 \mathrm{~h}$ preoperatively; median intra-abdominal pressure (IAP) and abdominal perfusion pressure (APP) were $22.5 \mathrm{mmHg}$ and $29 \mathrm{mmHg}$, respectively. After DL, IAP decreased and APP increased, both by an average of $60 \%$. Six patients survived, eight patients had a fatal outcome, resulting in a mortality of $57 \%$. An age under 1 year, weight under the 3rd percentile, an open abdomen treatment, an intestinal resection and an elevated serum lactate $>1.8 \mathrm{mmol} / \mathrm{L}$ were associated with an increased relative risk of death.

Conclusions Improving the outcome in pediatric patients with ACS by removing or attenuating risk factors is difficult. This emphasizes the need for early diagnosis and prompt DL once the diagnosis of ACS is made.
\end{abstract}

Keywords Abdominal compartment syndrome $\cdot$ Intra-abdominal pressure $\cdot$ Abdominal perfusion pressure $\cdot$ Decompressive laparotomy $\cdot$ Pediatrics

Electronic supplementary material The online version of this article (https://doi.org/10.1007/s00383-020-04632-0) contains supplementary material, which is available to authorized users.

Anthony di Natale

anthony.dinatale@kispi.uzh.ch

1 Department of Pediatric Surgery, University Children's Hospital Zurich, Zurich, Switzerland

2 Division of Immunology, University Children's Hospital Zurich, Zurich, Switzerland

3 Department of Pediatric and Neonatal Intensive Care, University Children's Hospital Zurich, Zurich, Switzerland

\section{Introduction}

Increased intra-abdominal pressure (IAP) can eventually lead to multi-organ failure. This condition has been termed abdominal compartment syndrome (ACS). It is associated with a very high morbidity and mortality.

Historically, the physiological effects of increased abdominal pressure on renal function and urinary output were first described by Wendt in 1876 [1]. The clinical significance became evident only in the early 1980s, after pediatric surgeons treating newborns with abdominal wall defects recognized a lethal impairment of the pulmonary and renal system by an increased intra-abdominal pressure [2]. Ever since, it has been shown that ACS leads to compression of the inferior vena cava, thereby reducing preload to the heart and having a detrimental effect on cardiovascular, respiratory, renal, and splanchnic organ function [3-6]. 
Various conditions can lead to the development of ACS, some of which are seemingly harmless at initial presentation and may not necessarily need surgery emergently [5, 7]. Although radiological modalities may show findings suggestive for ACS, definite diagnosis is still established by measurement of elevated IAP co-occurring with a new or worsening organ dysfunction [8]. The only effective treatment of ACS, once conservative therapies fail, is emergency decompressive laparotomy (DL) with open abdomen (OA) management [7, 9]. If left untreated, ACS results in a mortality of $100 \%$ [5].

In the past two decades, increased interest and research on ACS have led to a remarkable improvement in diagnosis and treatment of this clinical entity, especially in the adult population. Following the World Society of the Abdominal Compartment Syndrome's (WSACS) consensus on definitions, guidelines, and recommendations regarding the diagnosis and treatment of ACS, the mortality in adults due to ACS has decreased from 80 to $37 \%[10,11]$. However, among children with ACS, a high mortality of $40-85 \%$ still persists, which is partly attributable to the following conditions $[12,13]$; The scarcity of literature on ACS in the pediatric population shows that this patient group has been studied less extensively $[6,14]$. The etiology, risk factors, diagnosis and course of disease in the pediatric population differ from ACS in adults; hence, knowledge on ACS in adults does not directly translate to the pediatric population $[14,15]$. Also, recognition of ACS among pediatric health-care providers is still insufficient, as shown by conducted surveys $[16,17]$.

To further increase the awareness of ACS in the pediatric population, we aim to determine the incidence of ACS among patients who underwent an emergency laparotomy (EL) and to identify factors and disorders that predispose pediatric patients to ACS in a high-volume tertiary pediatric referral center. We also describe the effect of DL on clinical and laboratory parameters. Besides, for the purpose of making a better prediction on fatal outcome, we analyze variables and their association with mortality by comparing survivors and non-survivors diagnosed with ACS.

\section{Methods and materials}

\section{Patients' selection and ethics}

A retrospective chart review was conducted on all patients younger than 16 years of age who underwent EL between January 1, 2010, and December 31, 2018, at our tertiary pediatric referral center. Charts of patients diagnosed with ACS according to the latest definitions and guidelines of the WSACS were selected for further data collection as mentioned below [7]. Patients with a documented denial for the use of medical data were excluded.

The local Ethics Committee of Zurich approved the study protocol (BASEC Nr. 2018-01741).

\section{Definitions and data collection}

We defined ACS according to the latest WSACS consensus definition for ACS in the pediatric population: a sustained elevation in IAP of greater than $10 \mathrm{mmHg}$ associated with new or worsening organ dysfunction that can be attributed to elevated IAP [7]. A distinction is made between primary and secondary ACS. In general, primary ACS results from an injury or condition originating in the abdominopelvic region and frequently requires early surgery or an interventional radiological procedure. Secondary ACS develops as a result of an extra-abdominal process such as sepsis, capillary leak, or major burns, and is related to the required massive

Table 1 Distinction of primary and secondary ACS

Primary ACS

Reduced abdominal wall compliance

Increased intra-luminal contents

Increased intra-abdominal contents
Gastroschisis
Congenital diaphragmatic hernia
Small bowel intussusception
Ileus
Hirschsprung's disease
Bowel perforation
Peritonitis or intra-abdominal infection
Intra- or retroperitoneal bleeding
Ascites
Intra-abdominal tumor (Wilms', Burkitt's)
Acute pancreatitis
Abdominal organ transplantation

Shock (septic, cardiogenic, hemorrhagic) Burns

ACS abdominal compartment syndrome 
fluid resuscitation [18]. Table 1 summarizes some of the commonly associated pediatric conditions that predispose patients to ACS according to the WSACS definitions [7]. Retrospectively collected data of patients diagnosed with ACS included patient demographics, primary diagnosis, personal history, perioperative vital signs, preoperative support and outcome data: IAP, central venous pressure (CVP), mean arterial pressure (MAP), peak inspiratory pressure (PIP), oxygenation index $\left(\mathrm{OI}=\left(\mathrm{FiO}_{2} \times \mathrm{MAP}\right) / \mathrm{PaO}_{2}\right)$, need for vasopressors, diuretics, and dialysis, urine output, $\mathrm{OA}$ treatment, and death.

The Pediatric Index of Mortality 2 (PIM2) is a commonly used model for mortality prediction in children admitted to the intensive care unit (ICU). The risk of mortality is assessed using ten different patient variables collected on ICU admission [19, 20]. The PIM2 was used to assess illness severity.

Laboratory values collected included serum lactate, blood urea nitrogen (BUN), and serum creatinine. All variables were recorded $6 \mathrm{~h}$ before and $6 \mathrm{~h}$ after DL.

\section{Pressure monitoring}

IAP was measured according to the WSACS guidelines indirectly through a Foley bladder catheter. After instilling $1 \mathrm{~mL} / \mathrm{kg}$ sterile saline (with a minimum of $3 \mathrm{~mL}$ and a maximum of $25 \mathrm{~mL}$ ) in the bladder, the patient was placed in supine position if possible. The transducer was zeroed at the level of the symphysis. Values were obtained after $60 \mathrm{~s}$ of calibration at end expiration [7]. IAP measurements were repeated when clinically indicated, but at least every $4 \mathrm{~h}$.

Abdominal perfusion pressure (APP) was calculated using the formula MAP-IAP.

CVP was measured using the central venous line (CVL) and a transducer, which was zeroed at the level of the right atrium. Most commonly, the CVL was inserted via the right internal jugular vein. Patients with a femoral CVL were excluded for further CVP analysis due to potentially falsely elevated values.

\section{Statistical analysis}

Categorical variables were noted as numbers. Normal distribution of continuous variables was tested using the Shapiro-Wilk test. Normally distributed continuous variables were summarized as mean and standard deviation, while non-normally distributed values were summarized as median and value range. Univariate analysis of categorical variables was performed using two-tailed Fisher's exact test. Comparison of continuous variables was done using Mann-Whitney $U$ test. Statistical significance was defined as a $p$ value $<0.05$. Odds ratio and risk ratio were calculated along with a $95 \%$ confidence interval. All graphs and statistical analysis were created or performed with GraphPad Prism 6.00 for Mac (GraphPad Software, La Jolla California USA).

\section{Results}

In total, 1059 charts of patients who underwent laparotomy were reviewed, of whom 418 patients had EL. The most common indications for EL were intestinal obstruction $(n=171)$, followed by hollow organ perforation $(n=81)$ and intra-abdominal infection $(n=57)$. Fourteen patients ( 7 girls, 7 boys) had emergency DL for ACS, resulting in an incidence of ACS of 3.3\% of all EL. Ten of these 14 patients had primary ACS, mostly caused by intraperitoneal hemorrhage following percutaneous biopsy of the liver, removal of intra-abdominal dialysis catheter or kidney transplantation. There were two patients with intestinal perforations in our population that led to ACS. Two patients with omphalocele and gastroschisis, respectively, developed postoperative ACS after completed reduction. None was caused by a blunt abdominal trauma. Four patients had secondary ACS, mainly following septic shock $(n=3)$ and fluid resuscitation (Fig. 1).
Fig. 1 Etiology of abdominal compartment syndrome (ACS)

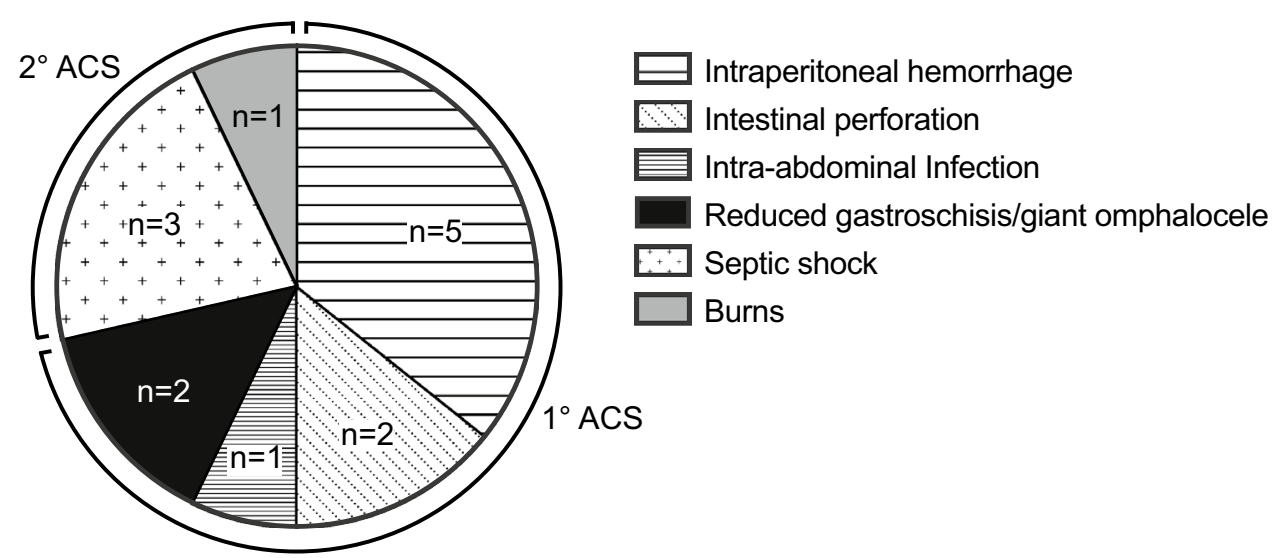


Table 2 Demographics and preoperative characteristics of all patients with ACS

\begin{tabular}{|c|c|}
\hline Patient characteristics & $\begin{array}{l}\text { All patients } \\
\text { with ACS } \\
n=14\end{array}$ \\
\hline \multicolumn{2}{|l|}{ Age } \\
\hline Mean \pm SD (years) & $1.1 \pm 1.7$ \\
\hline Median (range) (months) & $2(0.06-66)$ \\
\hline \multicolumn{2}{|l|}{ Sex } \\
\hline Male $(n)$ & 7 \\
\hline Female $(n)$ & 7 \\
\hline \multicolumn{2}{|l|}{ Weight } \\
\hline Median (range) $[\mathrm{kg}]$ & $4.1(1.1-18)$ \\
\hline$<3$ rd Percentile $(n)$ & 6 \\
\hline \multicolumn{2}{|l|}{ Preoperative support } \\
\hline Inotropic or Vasopressor $(n)$ & 10 \\
\hline Diuretics $(n)$ & 6 \\
\hline $\operatorname{ECMO}(n)$ & 2 \\
\hline Dialysis $(n)$ & 0 \\
\hline \multicolumn{2}{|l|}{ PIM2 score } \\
\hline Mean \pm SD $(\%)$ & $17 \pm 9.5$ \\
\hline Median (range) [\%] & $8.4(2.9-69)$ \\
\hline \multicolumn{2}{|l|}{ Preoperative parameters } \\
\hline Median IAP (range) [mmHg] & $22.5(14-43)$ \\
\hline Median MAP (range) $[\mathrm{mmHg}]$ & $52(28-79)$ \\
\hline Median APP (range) [mmHg] & $29(0-59)$ \\
\hline Median CVP (range) $[\mathrm{mmHg}]$ & $21.4(11-30)$ \\
\hline Median PIP (range) [mbar] & $28(19-40)$ \\
\hline Median OI (range) & $7.5(4.1-33)$ \\
\hline Median UO (range) $[\mathrm{mL} / \mathrm{kg} / \mathrm{h}]$ & $0.35(0-3.5)$ \\
\hline \multicolumn{2}{|l|}{ Preoperative laboratory values } \\
\hline Median serum lactate (range) $[\mathrm{mmol} / \mathrm{L}]$ & $3.8(0.7-14.6)$ \\
\hline Median BUN (range) [mmol/L] & $10(2.7-25)$ \\
\hline Median creatinine (range) $[\mu \mathrm{mol} / \mathrm{L}]$ & $51(27-154)$ \\
\hline
\end{tabular}

$A C S$ abdominal compartment syndrome, $A P P$ abdominal perfusion pressure, PIM2 Pediatric Index of Mortality 2, CVP central venous pressure, ECMO extracorporeal membrane oxygenation, $P I P$ peak inspiratory pressure, IAP intra-abdominal pressure, $O I$ oxygenation index, $M A P$ mean arterial pressure, $U O$ urine output, $B U N$ blood urea nitrogen

\section{Patient demographics and preoperative characteristics}

Median age at the time of ACS diagnosis was $1.1( \pm 1.7)$ years. Patients' weight at ICU admission ranged from 1.1 to $33 \mathrm{~kg}$. Six patients were below the $3 \mathrm{rd}$ weight percentile. Only one patient developed ACS on the regular ward following percutaneous liver biopsy with intraperitoneal hemorrhage, while all other patients were in the ICU before development of ACS. The mean predicted risk of mortality by the PIM2 score was $17 \% \pm 9.5$. Six hours preoperatively, median
IAP and APP were $22.5 \mathrm{mmHg}$ and $29 \mathrm{mmHg}$, respectively. Two patients were on extracorporeal membrane oxygenation support (ECMO). No patient required dialysis. All patients received nasogastric tube decompression, analgosedation, paralysis, and optimized fluid resuscitation. One patient received drainage of abdominal ascites without avoiding the development of ACS. Further patient demographics, preoperative support, vital signs, and laboratory values are shown in Table 2.

\section{Decompressive laparotomy}

As soon as diagnosis of ACS was established, indication for surgery was given and patients underwent emergency DL. All patients diagnosed with ACS had a midline laparotomy. Table 3 shows the effect of DL on patients' physiological parameters and laboratory values. Most of these improved or normalized after DL. Serum creatinine and BUN did not decrease within the first $6 \mathrm{~h}$ postoperatively. Although MAP only increased minimally, APP increased by an average of $60 \%(+18.5 \mathrm{mmHg})$ and IAP similarly decreased by an average of $60 \%(-14 \mathrm{mmHg})$. Ventilation improved, with PIP decreasing by an average of $19 \%$ ( $-5.3 \mathrm{mbar}$ ) and OI by $48 \%$ ( -6.7$)$. Serum lactate decreased by an average of $18 \%$ $(-0.8 \mathrm{mmol} / \mathrm{L})$ within the first $6 \mathrm{~h}$ postoperatively.

\section{Outcomes}

Six patients survived, whereas eight patients had a fatal outcome, resulting in a mortality of $57 \%$. Of all eight patients who have been diagnosed with ACS and deceased, five of them had a primary ACS caused by intraperitoneal hemorrhage $(n=2)$, intestinal perforation $(n=1)$, after completed reduction of a giant omphalocele $(n=1)$ and due to an intraabdominal infection $(n=1)$. Three patients died following secondary ACS due to sepsis $(n=2)$ and a severe burn injury $(n=1)$. The causes of death among these patients were multiple organ failure in five patients, intestinal ischemia and necrosis in one patient, respiratory failure in one patient, and cardiac failure in one patient.

Primary fascial closure was possible in six patients: four of them had ACS caused by an intraperitoneal hemorrhage, one patient following intestinal perforation, and one patient following intra-abdominal infection. Eight patients had OA treatment with negative pressure wound therapy. This included all four patients with secondary ACS, where no primary fascial closure was possible. At our institution, a V.A.C. ${ }^{\circledR}$ device (KCI, San Antonio TX, USA) was most commonly used for OA treatment. In these patients, abdominal wall closure was achieved after an average of 10.4 days.

Table 4 summarizes the individual demographics, preand postoperative characteristics and outcomes of each patient with ACS. The only statistically significant difference 
Table 3 Effect of decompressive laparotomy on physiological parameters and laboratory values $6 \mathrm{~h}$ pre- and postoperatively

\begin{tabular}{|c|c|c|c|c|}
\hline Parameters & Mean decrease & Median decrease & Range (min-max) & SD \\
\hline IAP (mmHg) & 14 & 14 & $3-31$ & \pm 8.1 \\
\hline CVP (mmHg) & 11.5 & 8.5 & $4-27$ & \pm 7.5 \\
\hline PIP (mbar) & 5.3 & 3 & $0-17$ & \pm 5.5 \\
\hline OI & 6.8 & 4.1 & $0-21.8$ & \pm 6.0 \\
\hline Parameters & Mean increase & Median increase & Range (min-max) & SD \\
\hline APP (mmHg) & 18.5 & 16 & $0-40$ & \pm 12.8 \\
\hline MAP (mmHg) & 3.4 & 0 & -10 to 22 & \pm 10 \\
\hline $\mathrm{UO}(\mathrm{mL} / \mathrm{kg} / \mathrm{h})$ & 3.0 & 3.3 & $0.4-6.3$ & \pm 1.5 \\
\hline Laboratory values & Mean decrease & Median decrease & Range (min-max) & SD \\
\hline Serum lactate $(\mathrm{mmol} / \mathrm{L})$ & 0.8 & 0.3 & $0-4.6$ & \pm 2 \\
\hline $\mathrm{BUN}(\mathrm{mmol} / \mathrm{L})$ & 0 & 0 & -3.9 to 4.5 & \pm 2 \\
\hline Serum creatinine $(\mu \mathrm{mol} / \mathrm{L})$ & 0 & 0 & -40 to 42 & \pm 21.5 \\
\hline
\end{tabular}

$I A P$ intra-abdominal pressure, $A P P$ abdominal perfusion pressure, $C V P$ central venous pressure, $M A P$ mean arterial pressure, $P I P$ peak inspiratory pressure, $U O$ urine output, $O I$ oxygenation index, $B U N$ blood urea nitrogen

between survivors and non-survivors among patients with ACS was a preoperatively elevated BUN, as presented in Appendix 1.

As shown in Table 5, the age $<1$ year (RR 2.33, 95\% CI $0.66-8.21 ; p 0.58$ ), weight under the 3rd percentile (RR $1.33,95 \%$ CI $0.54-3.26 ; p 0.63$ ), OA treatment (RR 2.25, 95\% CI 0.68-7.47; $p 0.28$ ), intestinal resection (RR 1.5, 95\% CI $0.64-3.47 ; p 0.58$ ), elevated preoperative serum lactate (RR 1.91, 95\% CI 0.36-10.0; $p 0.38$ ), and elevated postoperative serum lactate (RR 3.5, 95\% CI 0.58-21.1; $p 0.24$ ) were associated, although not statistically significant, with an increased relative risk of death.

\section{Discussion}

ACS is a well-known and described, and often life-threatening condition. Despite the efforts and dedication of the WSACS to raise awareness of ACS, it still carries a high morbidity and mortality, especially in the pediatric population. Most of the pediatric guidelines regarding ACS are based on adults, due to the lack of multi-center prospective studies in children.

Over a 9-year period at our tertiary pediatric center, 418 emergency laparotomies were performed. Of these, 14 were emergency DL in patients with ACS, thus resulting in an incidence of $3.3 \%$ of all EL. This is consistent with the reported incidence of pediatric ACS between $0.6 \%$ and $9.8 \%$ in the scarce pediatric literature $[5,12,13,15,21,22]$.

Despite surgical decompression, eight of our patients died. This overall mortality rate of $57 \%$ in patients with ACS is similar to other recent publications which reported mortality rates between 40 and $60 \%$ [12, 22-24].
The incidence and frequency of ACS in adults has been underestimated for many years. This could be explained by the low awareness for ACS despite intensified and deepened research, implemented guidelines, and training of physicians and nurses [25-27]. These factors partly also apply to the pediatric population as conducted surveys among pediatric health-care providers indicate $[16,17]$. Many pediatric intensivists and surgeons are aware of ACS, but knowledge on published consensus definitions, measurement techniques, and, most of all, on management are likely insufficient and not applied throughout [26]. It is also possible that infants and children may better compensate increased abdominal tension with a more compliant abdominal wall [15]. Hence, timely diagnosis and therapy of ACS may be delayed and therefore contribute to the still unacceptably high mortality of ACS in children.

Furthermore, risk factors and conditions predisposing pediatric patients to ACS differ markedly when compared to the adult population. While ruptured aortic aneurysm, hepatic failure, and severe pancreatitis are commonly reported in adult patients with ACS, they have little to no significance in the pediatric population [28].

Primary ACS was more common than secondary ACS in our study. The most common conditions leading to ACS were intraperitoneal hemorrhage and intestinal perforation with peritonitis. Hence, conditions predisposing to ACS in our population, were mostly associated with an increase in abdominal content (e.g., intraperitoneal hemorrhage and bowel perforation). This corresponds to the findings of other groups [15, 22].

The median IAP in our patients diagnosed with ACS was $22.5 \mathrm{mmHg}(14-43 \mathrm{mmHg})$, with an average decrease of $60 \%$ after DL to a median IAP of $9 \mathrm{mmHg}$. As mentioned 


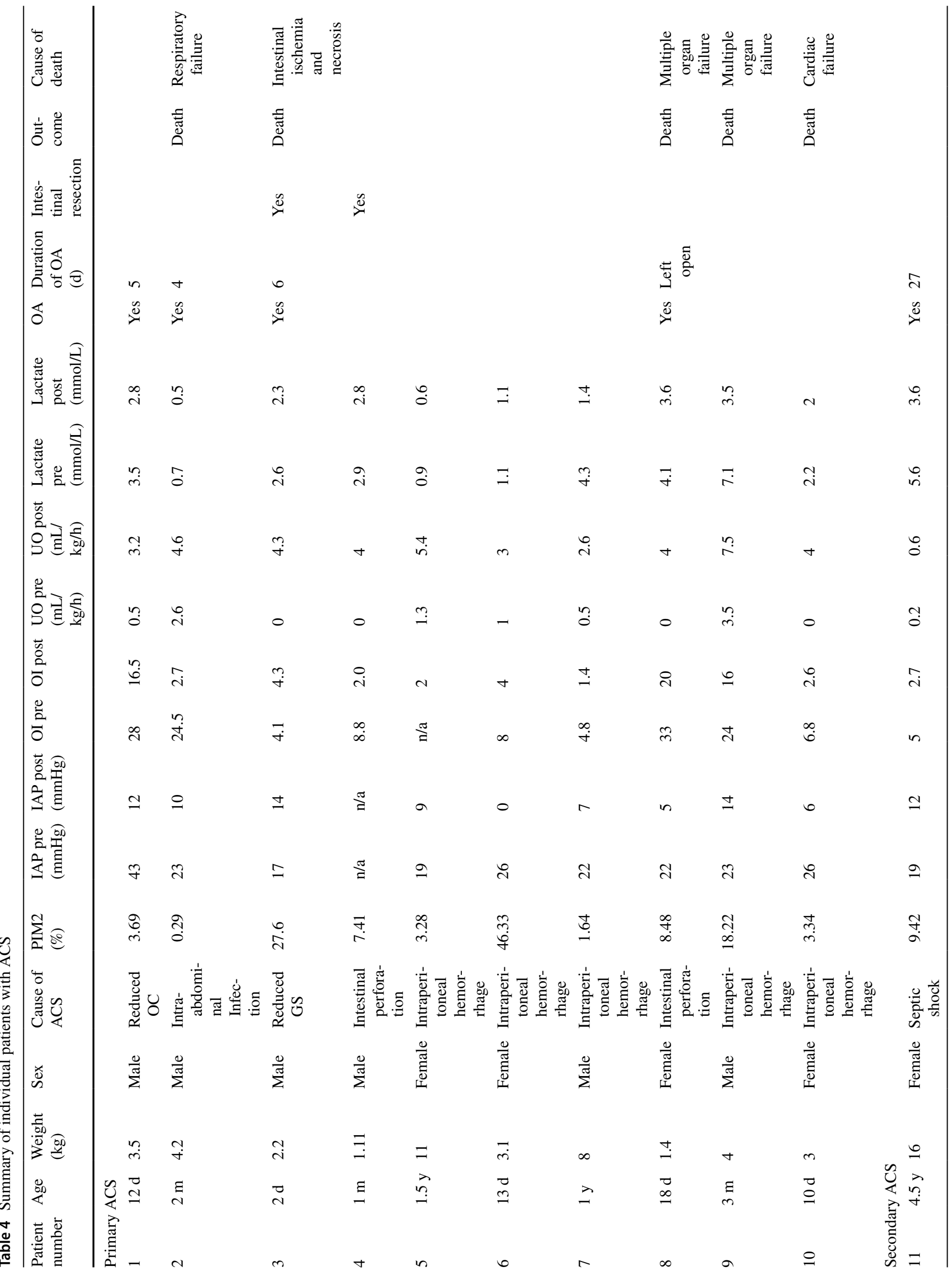




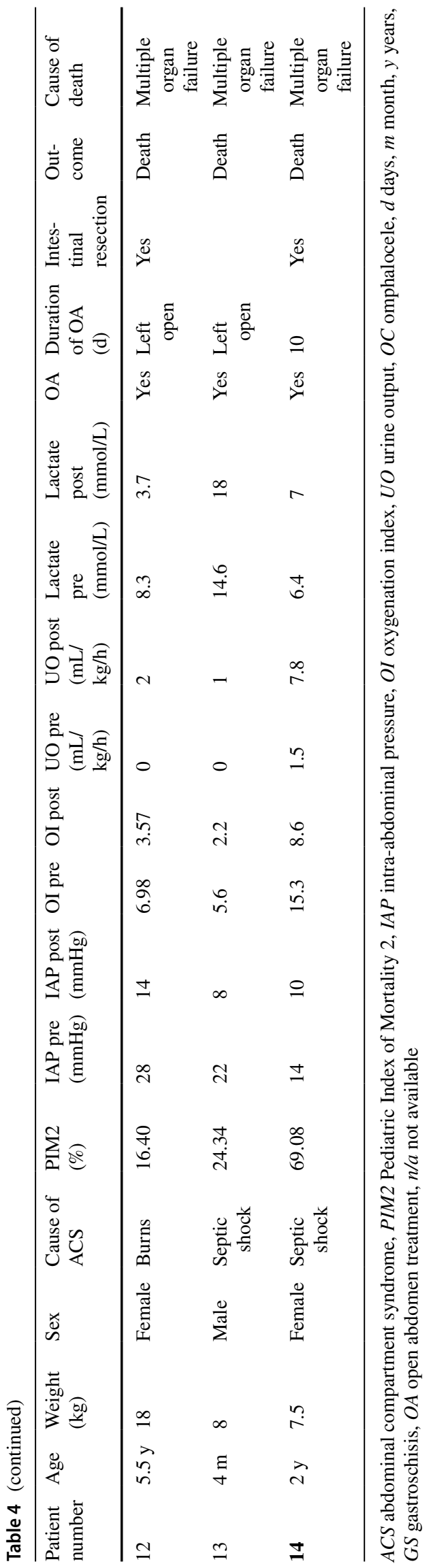

above, according to the WSACS guidelines, pediatric ACS is defined as a sustained elevation in IAP of greater than $10 \mathrm{mmHg}$ associated with a new onset of organ dysfunction or failure. For adults, the threshold is $20 \mathrm{mmHg}$ with or without an APP of less than $60 \mathrm{mmHg}$ [7]. However, there are no normal IAP pressure values for healthy children. Ejike et al. established that baseline IAP in critically ill children is approximately $4-10 \mathrm{mmHg}$. Cheatham et al. demonstrated that keeping APP $>50 \mathrm{mmHg}$ significantly improved morbidity and mortality in adults [29]. There is currently no recommendation on what the appropriate APP as a resuscitation goal for children is. We assume that children would tolerate a lower APP than adults since their MAP is lower. To our knowledge, there is only one other publication, by Horoz et al. that looked at APP in children. They determined a cut-off point of $53 \mathrm{mmHg}$ and showed that a decrease in APP below $53 \mathrm{mmHg}$ was associated with increased mortality [24]. We were not able to show an increased mortality with an APP $<53 \mathrm{mmHg}$ in our patients. Surviving patients had a median APP of $23 \mathrm{mmHg}$ before and $44.5 \mathrm{mmHg}$ after DL. Thus we cannot draw the same conclusion from our data as Horoz et al. Yet, APP could be an additional parameter to evaluate pediatric patients with ACS and help to detect and eventually avert ACS.

However, treatment strategies should mainly focus on attenuating IAP instead of increasing MAP. There are well described non-surgical strategies to decrease IAP: evacuation of intra-luminal content (e.g., nasogastric and rectal tubes), evacuation of intra-abdominal space occupying material (e.g., ascites), improving abdominal wall compliance (sedation, paralysis), optimizing fluid administration, and systemic perfusion (e.g., goal-directed fluid resuscitation, hemodialysis) [7]. In our study, all patients received nasogastric tube decompression, analgosedation, paralysis, and optimized fluid resuscitation. One patient received drainage of abdominal ascites, but this could not avoid ACS and DL. This emphasizes that clinicians should be careful not to overestimate these measures in their effectiveness.

DL is still considered the standard of care in patients with ACS, as it is the only available effective treatment. Its efficacy has previously been shown by other authors and is reflected also by our results [22,23]. Prior to DL, all patients with ACS were oliguric, on high ventilator support, and showed signs of splanchnic hypoperfusion and impaired renal function. Following DL, physiologic parameters and laboratory values improved, as a consequence of increased cardiac preload and restored organ perfusion. Nonetheless, recuperation of organ dysfunction after DL for ACS is variable. Although the average urinary output increased after DL, serum creatinine and BUN did not decrease, as shown in Table 3. On the one hand, this could be explained by the fact that an increased urinary output is not necessarily 
Table 5 Univariate analysis of variables associated with mortality

\begin{tabular}{|c|c|c|c|c|}
\hline Variables & $\begin{array}{l}\text { Deaths } / n \\
\text { patients }\end{array}$ & $\mathrm{RR}$ & OR & $p$ value \\
\hline \multicolumn{5}{|l|}{ Age } \\
\hline Age $<1$ year & $6 / 9$ & 2.33 & 3 & \multirow[t]{2}{*}{0.58} \\
\hline Age $>1$ year & $2 / 5$ & CI 95\% $(0.66,8.21)$ & CI 95\% $(0.31,28.84)$ & \\
\hline \multicolumn{5}{|c|}{ Weight $<3$ rd percentile } \\
\hline Yes & $4 / 6$ & 1.33 & 2 & \multirow[t]{2}{*}{0.63} \\
\hline No & $4 / 8$ & CI 95\% $(0.54,3.26)$ & CI 95\% $(0.22,17.89)$ & \\
\hline \multicolumn{5}{|l|}{ OA treatment } \\
\hline Yes & $6 / 8$ & 2.25 & 6 & \multirow[t]{2}{*}{0.28} \\
\hline No & $2 / 6$ & CI 95\% $(0.68,7.47)$ & CI 95\% $(0.58,61.84)$ & \\
\hline \multicolumn{5}{|c|}{ Intestinal resection } \\
\hline Yes & $3 / 4$ & 1.5 & 3 & \multirow[t]{2}{*}{0.58} \\
\hline No & $5 / 10$ & CI 95\% $(0.64,3.47)$ & CI 95\% $(0.23,39.61)$ & \\
\hline \multicolumn{5}{|c|}{ Serum lactate $>1.8 \mathrm{mmol} / \mathrm{L}$ preoperatively } \\
\hline Yes & $7 / 11$ & 1.91 & 3.5 & \multirow[t]{2}{*}{0.38} \\
\hline No & $1 / 3$ & CI 95\% $(0.36,10.0)$ & CI $95 \%(0.23,51.9)$ & \\
\hline \multicolumn{5}{|c|}{ Serum lactate $>1.8 \mathrm{mmol} / \mathrm{L}$ postoperatively } \\
\hline Yes & $7 / 10$ & 3.5 & 7 & \multirow[t]{2}{*}{0.24} \\
\hline No & $1 / 4$ & CI 95\% $(0.58,21.1)$ & CI 95\% $(0.5,97.7)$ & \\
\hline
\end{tabular}

$O A$ open abdomen, $R R$ relative risk, $O R$ odds ratio, $C I$ confidence interval correlated to an increased glomerular filtration rate (GFR), on the other hand it could also be attributed to the short time period of only $6 \mathrm{~h}$ after DL when serum creatinine and BUN were measured in our study.

We emphasize that, although IAP is consistently lower after decompression, the overall mortality in pediatric patients with ACS remains high [22, 30]. Comparing the characteristics and parameters of survivors and nonsurvivors, preoperative BUN was significantly higher in non-survivors. The elevated BUN in non-survivors may reflect a longer kidney hypoperfusion as a consequence of potentially delayed ACS diagnosis. As manifest, ACS affects all organ systems, prolonged end organ hypoperfusion, and non-oxygenation causes irreversible cellular damage that does not recover after DL. Hence, delayed diagnosis and therapy worsens outcome significantly and is a relevant contributor to the continued high mortality of ACS in the pediatric population.

We analyzed several variables and their association with mortality to make a better prediction on mortality. Because of the inherent limitations of this study-being retrospective, single-centered, and having a relatively small sample size-it is not possible to make final recommendations on which variables have a stronger association with mortality. But these variables: an age under 1 year, weight under the 3rd percentile, an OA treatment, an intestinal resection as well as an elevated pre- or postoperative serum lactate $>1.8 \mathrm{mmol} / \mathrm{L}$ were all associated with an increased relative risk of death, although not statistically significant.
Improving the outcome in these patients by removing or attenuating these factors seems difficult, as many of the variables are given and not readily influenceable. To make a definitive statement on the association of these variables with mortality, prospective studies on a larger patient cohort are needed. Nevertheless, we think that the abovementioned variables must be considered in the evaluation and management of pediatric patients with ACS.

We conclude that, despite improved intensive care and raised awareness, ACS in children is still associated with an unacceptably high mortality. This emphasizes the need for early diagnosis and prompt treatment to improve patients' outcomes. Given the fact that the incidence of ACS is fairly low, clinicians should maintain a high level of suspicion and measure IAP early and regularly in patients at risk. Once the diagnosis of ACS is suspected or made, emergency DL is imperative.

Funding The authors hereby confirm that no funding was received for this project.

\section{Compliance with ethical standards}

Conflict of interest Anthony di Natale, Ueli Moehrlen, Hannah Rachel Neeser, Noëmi Zweifel, Martin Meuli, Andrea Alexis Mauracher, Barbara Brotschi, and Sasha Job Tharakan hereby confirm that no conflicts of interest exist. 
Ethical approval All procedures performed in studies involving human participants were in accordance with the ethical standards of the institutional and cantonal research committee (The local Ethics Committee of Zurich, BASEC Nr. 2018-01741) and with the 1964 Helsinki Declaration and its later amendments or comparable ethical standards.

Informed consent The authors hereby confirm that informed consent was obtained from all individual patients included in this study.

\section{References}

1. Wendt E (1876) Ueber den Einfluss des intraabdominalen Druckes auf die Absonderungsgeschwindigkeit des Harnes. Arch Heilkunde 17:527-546

2. Wesley JR, Drongowski R, Coran AG (1981) Intragastric pressure measurement: a guide for reduction and closure of the silastic chimney in omphalocele and gastroschisis. J Pediatr Surg $16: 264-270$

3. Kron IL, Harman PK, Nolan SP (1984) The measurement of intraabdominal pressure as a criterion for abdominal re-exploration. Ann Surg 199:28-30

4. Cullen DJ, Coyle JP, Teplick R, Long MC (1989) Cardiovascular, pulmonary, and renal effects of massively increased intraabdominal pressure in critically ill patients. Crit Care Med $17: 118-121$

5. Ejike JC, Humbert S, Bahjri K, Mathur M (2007) Outcomes of children with abdominal compartment syndrome. Acta Clin Belg 62:141-148

6. Thabet FC, Ejike JC (2017) Intra-abdominal hypertension and abdominal compartment syndrome in pediatrics. A Rev J Crit Care 41:275-282

7. Kirkpatrick AW, Roberts DJ, De Waele JJ, Jaeschke R, Malbrain MLNG et al (2013) The pediatric guidelines sub-committee for the world society of the abdominal compartment syndrome, intra-abdominal hypertension and the abdominal compartment syndrome: updated consensus definitions and clinical practice guidelines from the world society of the abdominal compartment syndrome. Intensiv Care Med 39:1190-1206

8. Je BK, Kim HK, Horn PS (2019) Abdominal compartment syndrome in children: clinical and imaging features. Am J Roentgenol 212:655-664

9. Meldrum DR, Moore FA, Moore EE, Franciose RJ, Sauaia A, Burch JM (1997) Prospective characterization and selective management of the abdominal compartment syndrome. Am J Surg 174:667-673

10. De Waele JJ, Pletinckx P, Blot S, Hoste E (2006) Saline volume in transvesical intra-abdominal pressure measurement: enough is enough. Intensiv Care Med 32:455-459

11. Carr JA (2013) Abdominal compartment syndrome: a decade of progress. J Am Coll Surg 216:135-146

12. Thabet FC, Bougmiza IM, Chehab MS, Bafaqih HA, Al Mohaimeed SA, Malbrain MLNG (2015) Incidence, risk factors, and prognosis of intra-abdominal hypertension in critically Ill children. J Intensiv Care Med 31:403-408

13. Diaz FJ, Fernandez Sein A, Gotay F (2006) Identification and management of abdominal compartment syndrome in the pediatric intensive care unit. P R Health Sci J 25:17-22

14. De Waele JJ, Ejike JC, Leppäniemi A, De Keulenaer BL, Kirkpatrick AW, Roberts DJ, Malbrain MLNG (2015) Intra-abdominal hypertension and abdominal compartment syndrome in pancreatitis, paediatrics, and trauma. Anaesthesiol Intensiv Ther 47:219-227

15. Beck R, Halberthal M, Zonis Z, Shoshani G, Hayari L, Bar-Joseph $\mathrm{G}$ (2001) Abdominal compartment syndrome in children. Pediatr Crit Care Med 2:51-56

16. Ejike JC, Newcombe J, Baerg J et al (2010) Understanding of abdominal compartment syndrome among pediatric healthcare providers. Crit Care Res Pract. https://doi.org/10.1155/2010/87601 3

17. Kaussen T, Steinau G, Srinivasan PK et al (2012) Recognition and management of abdominal compartment syndrome among German pediatric intensivists: results of a national survey. Ann Intensiv Care 2:S8

18. Malbrain MLNG, Cheatham ML, Kirkpatrick A, Sugrue M, Parr M, De Waele JJ et al (2006) Results from the international conference of experts on intra-abdominal hypertension and abdominal compartment syndrome. I. Definitions. Intensiv Care Med 32:1722-1732

19. Slater A, Shann F, Pearson G et al (2003) PIM2: a revised version of the paediatric index of mortality. Intensiv Care Med 29:278-285

20. Slater A, Shann F (2004) The suitability of the pediatric Index of mortality (PIM), PIM2, the pediatric risk of mortality (PRISM), and PRISM III for monitoring the quality of pediatric intensive care in Australia and New Zealand. Pediatr Crit Care Med 5:447-453

21. Divarci E, Karapinar B, Yalaz M, Ergun O, Celik A (2016) Incidence and prognosis of intraabdominal hypertension and abdominal compartment syndrome in children. J Pediatr Surg 51:503-507

22. Pearson EG, Rollins MD, Vogler SA, Mills MK, Lehman EL, Jacques E et al (2010) Decompressive laparotomy for abdominal compartment syndrome in children: before it is too late. J Pediatr Surg 45:1324-1329

23. Thomas S, Kriplani D, Crane C, Dehom SO, Oei G, Baerg J et al (2017) Outcomes in pediatric patients with abdominal compartment syndrome following urgent exploratory laparotomy. J Pediatr Surg 52:1144-1147

24. Horoz OO, Yildizdas D, Sari Y, Unal I, Ekinci F, Petmezci E (2019) The relationship of abdominal perfusion pressure with mortality in critically ill pediatric patients. J Pediatr Surg 1-5

25. Newcombe J, Mathur M, Ejike JC (2012) Abdominal compartment syndrome in children. Crit Care Nurse 32:51-61

26. Wise R, Roberts DJ, Vandervelden S, Debergh D, De Waele JJ, De laet I et al (2015) Awareness and knowledge of intra-abdominal hypertension and abdominal compartment syndrome: results of an international survey. Anaesthesiol Intensiv Ther 47:14-29

27. Zhang H-Y, Liu D, Tang H, Sun S-J, Ai S-M, Yang W-Q et al (2016) Study of intra-abdominal hypertension prevalence and awareness level among experienced ICU medical staff. Mil Med Res 3:1-7

28. Holodinsky JK, Roberts DJ, Critical CB (2013) Risk factors for intra-abdominal hypertension and abdominal compartment syndrome among adult intensive care unit patients: a systematic review and meta-analysis. Crit Care 17:R249

29. Cheatham ML, White MW, Sagraves SG, Johnson JL, Block EF (2000) Abdominal perfusion pressure: a superior parameter in the assessment of intra-abdominal hypertension. J Trauma 49:621-627

30. De Waele JJ, Hoste EA, Malbrain ML (2006) Decompressive laparotomy for abdominal compartment syndrome-a critical analysis. Crit Care 10:R51

Publisher's Note Springer Nature remains neutral with regard to jurisdictional claims in published maps and institutional affiliations. 